ARTICLE

\title{
Disentangling the recognition complexity of a protein hub using a nanopore
}

\author{
Lauren Ashley Mayse (10 1,2, Ali Imran¹, Motahareh Ghahari Larimi ${ }^{1,3}$, Michael S. Cosgrove ${ }^{4}$,
} Aaron James Wolfe ${ }^{1,5,6,7}$ \& Liviu Movileanu (1) 1,2,8凶

WD40 repeat proteins are frequently involved in processing cell signaling and scaffolding large multi-subunit machineries. Despite their significance in physiological and disease-like conditions, their reversible interactions with other proteins remain modestly examined. Here, we show the development and validation of a protein nanopore for the detection and quantification of WD40 repeat protein 5 (WDR5), a chromatin-associated hub involved in epigenetic regulation of histone methylation. Our nanopore sensor is equipped with a 14residue Win motif of mixed lineage leukemia 4 methyltransferase $\left(M L L 4_{\text {Win }}\right.$ ), a WDR5 ligand. Our approach reveals a broad dynamic range of MLL4 Win WDR5 interactions and three distant subpopulations of binding events, representing three modes of protein recognition. The three binding events are confirmed as specific interactions using a weakly binding WDR5 derivative and various environmental contexts. These outcomes demonstrate the substantial sensitivity of our nanopore sensor, which can be utilized in protein analytics.

\footnotetext{
${ }^{1}$ Department of Physics, Syracuse University, 201 Physics Building, Syracuse, NY 13244-1130, USA. ${ }^{2}$ Department of Biomedical and Chemical Engineering, Syracuse University, 329 Link Hall, Syracuse, NY 13244, USA. ${ }^{3}$ Section on Molecular Transport, Eunice Kennedy Shriver National Institute of Child Health and Human Development, National Institutes of Health, Bethesda, MD 20892, USA. ${ }^{4}$ Department of Biochemistry and Molecular Biology, State University of New York - Upstate Medical University, 4249 Weiskotten Hall, 766 Irving Avenue, Syracuse, NY 13210, USA. ${ }^{5}$ Ichor Life Sciences, Inc, 2651 US Route 11, LaFayette, NY 13084, USA. ${ }^{6}$ Lewis School of Health Sciences, Clarkson University, 8 Clarkson Avenue, Potsdam, NY 13699, USA. ${ }^{7}$ Department of Chemistry, State University of New York, College of Environmental Science and Forestry, 1 Forestry Dr, Syracuse, NY 13210, USA. ${ }^{8}$ The Biolnspired Institute, Syracuse University, Syracuse, NY 13244, USA. 凶email: Imovilea@syr.edu
} 
etailed knowledge of the human genome has stimulated the discovery of over 360 WD40 repeat proteins $(\text { WDRs })^{1,2}$. WDRs are versatile in mediating numerous protein-protein interactions (PPIs) across diverse cellular pathways and play a pivotal regulatory role in scaffolding enzymatic complexes $^{3,4}$. Although they are among the most frequently encountered PPI domains in the human ${ }^{5,6}$ proteome, selective and dynamic interactions of WDRs with dozens of protein substrates are still ambiguous. Furthermore, major perturbations in their physical associations with other proteins can lead to pathological conditions through diverse disease-associated signaling mechanisms ${ }^{7}$. However, it is difficult to appraise the transient nature of these interactions using existing methods due to their restricted time resolution as well as their inability to identify and characterize the heterogeneity of binding specifics ${ }^{8}$. Biological and synthetic nanopores have served as a powerful tool for sampling reversible protein-peptide ${ }^{9,10}$ and protein-protein ${ }^{11-14}$ interactions in solution. Advantages of nanopores using the resistive-pulse technique ${ }^{15}$ include the ability to explore a wide spectrum of kinetic and affinity constants due to an expanded time bandwidth. In addition, nanopore sensors feature the potential for integration into high-throughput technologies ${ }^{16-18}$ and for quantitative determinations in challenging heterogenous solutions ${ }^{12,13,19-21}$. Moreover, these are real-time and label-free measurements that employ selective sensing elements, which are modifiable with atomic precision ${ }^{22-27}$.

The primary challenge in detecting WDRs using a nanopore is the complexity of the interaction. WDRs are too large to enter the nanopore, so these interactions must be probed outside the lumen. Previously, a monomeric $\beta$-barrel scaffold of ferric hydroxamate uptake component A (FhuA) ${ }^{28}$ from Escherichia coli, named tFhuA, has been used to overcome this challenge ${ }^{12,13}$. A protein ligand was fused to tFhuA via a flexible Gly/Ser-rich hexapeptide tether and a peptide adaptor was attached to the $\mathrm{N}$ terminus of ligand to detect protein-protein interactions without steric hindrance ${ }^{12}$. Furthermore, the binding interface between most WDRs and their protein partners are not mediated by a large and relatively flat surface. For example, the 334-residue WD40 repeat protein 5 (WDR5) has a seven-bladed, WD40 repeat-based $\beta$ propeller circular structure, surrounding a central cavity ${ }^{4,29}$. A segment of the inner lining of this cavity serves as the binding site for the WDR5 interaction sequence (Win) motif of human mixed lineage leukemia (MLL/ SET1) methyltrasferases ${ }^{30-33}$. This is also named the Win binding site. A Win motif must enter the WDR5 cavity to reach the Win binding site $\mathrm{s}^{32,33}$.

In this work, we are able to overcome these arduous challenges and disentangle a multimodal protein recognition process using an engineered protein nanopore. Our tFhuA nanopore is fused to a 14-residue Win motif of mixed lineage leukemia 4 (MLL4 $\left.4_{\text {Win }}\right)$ methyltransferase (Fig. 1a), a WDR5 ligand. This nanopore fusion protein, named $\mathrm{MLL} 4_{\mathrm{Win}} \mathrm{tFhuA}$, also includes a peptide adaptor at its $\mathrm{N}$ terminus. We demonstrate that $\mathrm{MLL} 4_{\mathrm{Win}} \mathrm{t}$ FhuA detects WDR5 with single-molecule fidelity in solution. MLL4 ${ }_{\text {Win }}$ must enter the WDR5 cavity, which has a conical geometry with a maximum internal diameter of $\sim 15 \AA$, as measured from side chain to side chain (Fig. 1b). Once MLL4 $4_{\text {Win }}$ partitions $\sim 11 \AA$ into the WDR5 cavity, several interactions are coordinated by an evolutionarily conserved Arg residue at position $\mathrm{P}_{0}$ of MLL4 $4_{\text {Win }}$ and adjacent side chains of the Win binding site (Supplementary Fig. 1 and Tables 1,2 ). We show an unusually large range of kinetics with distinct distributions relating to subpopulations of the transiently formed MLL4 ${ }_{\mathrm{Win}}$-WDR5 complexes. Biolayer interferometry (BLI) confirms the lower limit of the association rate constant for these complex interactions, yet it is unable to detect multiple binding subpopulations. We also use two WDR5 derivatives and various experimental conditions to validate these multimodal protein recognition events.

\section{Results and discussion}

A nanopore sensor reveals three distant binding events. At a transmembrane potential of $-20 \mathrm{mV}$, a relatively quiet singlechannel current was recorded with a single MLL4 ${ }_{\text {Win }}$ tFhuA nanopore (Fig. 2a). The presence of WDR5 in the cis compartment at nanomolar concentrations produced infrequent current blockades (Supplementary Fig. 2 Table 3), likely because of the entropic penalty of MLL4 $4_{\text {Win }}$ to partition into the WDR5 cavity. These current blockades occurred as WDR5 was held in the proximity of the pore opening during its reversible captures by $M L L 4_{\text {win }}$, obstructing a fraction of the ionic flux through the nanopore. They were not noted when an unmodified tFhuA nanopore $^{12,13}$, without the MLL4 ${ }_{\text {Win }}$ ligand, was exposed to WDR5 added to the cis compartment. However, very rare and brief current spikes were observed, likely due to collisions of WDR5 with the opening of the unmodified tFhuA nanopore (Supplementary Figs. 3, 4). Taken together, these findings indicate that WDR5 did not produce significant current blockades due to nonspecific interactions with the cis opening of the nanopore. The lack of current blockades without MLL4 $4_{\text {win }}$ shows that the ligand must first bind to WDR5 to enable WDR5-produced current blockades.

WDR5-released and WDR5-captured events recorded with MLL4 $4_{\text {Win }}$ tFhuA corresponded to the open-substate, $\mathrm{O}_{\mathrm{on}}$, and closed-substate, $\mathrm{O}_{\text {off }}$, respectively. WDR5-captured events were noted in a concentration-dependent manner when WDR5 was added to the cis compartment (Fig. 2b-d; Supplementary Fig. 5). These single-channel electrical traces were low-pass filtered at a frequency of $100 \mathrm{~Hz}$ using an 8-pole Bessel filter. Yet, WDR5captured events were not detectable when WDR5 was added to the trans compartment (Supplementary Fig. 6). This result agrees with our previous studies ${ }^{12,13,34}$, which showed that tFhuA and its derivatives insert into the membrane with a single orientation. Moreover, WDR5-captured events were not detectable at a positive transmembrane potential of $+20 \mathrm{mV}$ (Supplementary Fig. 7). This outcome is in accord with the observation of current blockades produced by the positively charged WDR5 at a negative transmembrane potential (e.g., $\mathrm{pI}_{\mathrm{WDR} 5}=8.27$ ).

Surprisingly, we noted an extensive spectrum of WDR5captured durations, between $\sim 3 \mathrm{~ms}$ and $\sim 40 \mathrm{~s}$. This result stimulated detailed statistical analyses of both the WDR5released and WDR5-captured durations, whose mean values were denoted by $\tau_{\text {on }}$ and $\tau_{\text {off }}$, respectively. To identify potentially distinct subpopulations of these durations, we employed the maximum likelihood method ${ }^{35,36}$ and logarithm likelihood ratio (LLR) tests ${ }^{37-39}$ to determine the most accurate model of these time constants. Durations of WDR5-released events showed a single-exponential distribution (Fig. 2e). Remarkably, the best model for WDR5-captured durations was a three-exponential distribution (Fig. 2f). Fits to a single-, a two-, or a fourexponential model were not better, as judged by the LLR value. The three WDR5-captured durations were almost one order of magnitude apart from each other. For example, the mean durations of WDR5-captured events were $\sim 12 \mathrm{~ms}, \sim 120 \mathrm{~ms}$, and $\sim 1370 \mathrm{~ms}$ at $1 \mu \mathrm{M}$ WDR5 (Supplementary Table 4 ). We called these short-, medium-, and long-lived events, respectively. Event probabilities of these WDR5-captured events, $P_{1}, P_{2}$, and $P_{3}$, respectively, were independent of WDR5 concentration, [WDR5], and followed the inequality: $P_{1}>P_{2}>P_{3}$ (Supplementary Table 5).

Moreover, the mean normalized amplitude of WDR5produced current blockades, $\left(I / I_{0}\right)$, was independent of [WDR5] ( $n=5$ independently reconstituted nanopores; Fig. 2g; Supplementary Table 6). Here, $I_{0}$ and $I$ denote the single-channel currents of the WDR5-released substate of MLL4 $4_{\text {Win }}$ tFhuA and the amplitude of WDR5-produced current blockades, respectively. Histograms of the normalized amplitude of WDR5- 


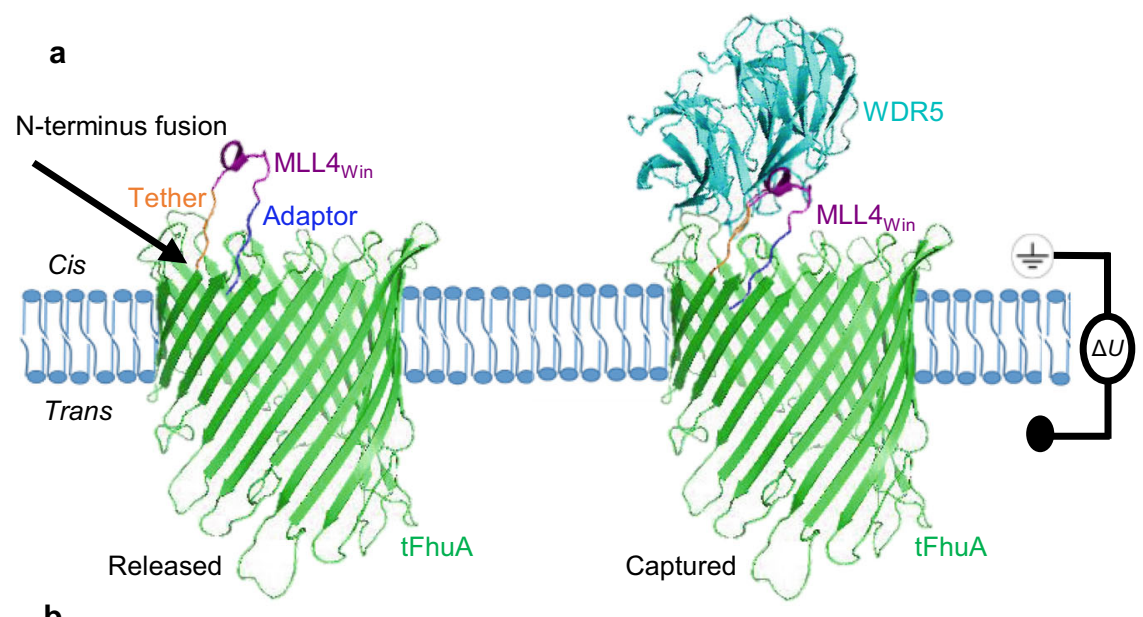

b
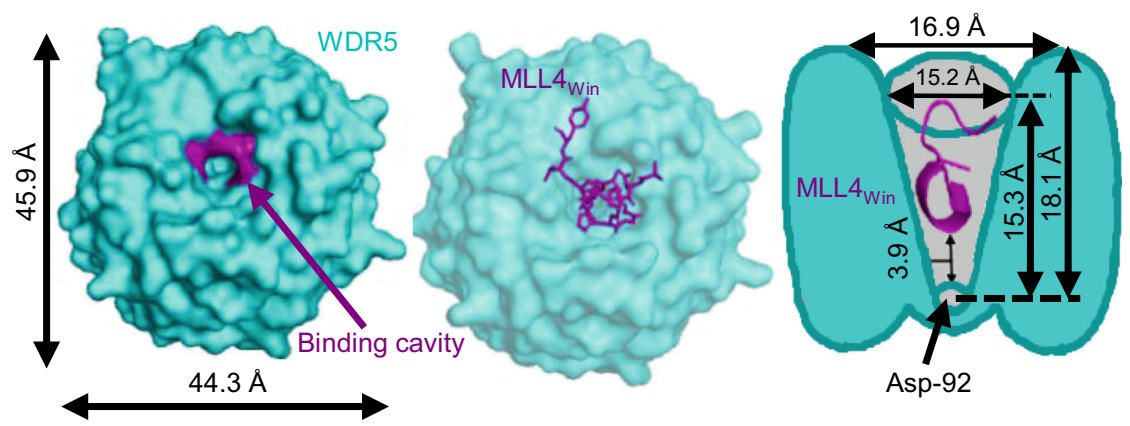

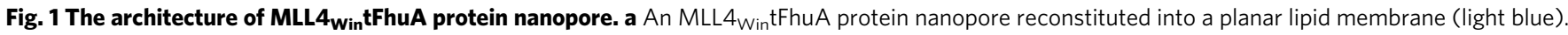
This protein comprises a tFhuA protein nanopore (green), a flexible Gly/Ser-rich hexapeptide tether (yellow), a 14-residue MLL4win peptide ligand (magenta), and a peptide adaptor (blue). This model was developed using Protein Data Bank files 1BY3 (FhuA) ${ }^{28}$ and 4ERZ (MLL4 Win - WDR5) ${ }^{32}$. The leftand right-hand cartoons show the protein nanopore in the released and WDR5-captured substates, respectively. WDR5 is marked in cyan. The cis compartment of the measurement chamber is grounded. A transmembrane potential, $\Delta U$, is applied. The black arrow indicates the $N$ terminus of tFhuA that is fused to MLL4 ${ }_{\text {Win }}$ via the flexible tether. $\mathbf{b}$ On the left side, a top-view cartoon of WDR5 (in cyan) shows the binding cavity. Phe-133, Cys-261, and Ser-91 are colored in magenta. The central cartoon is the WDR5-MLL4 ${ }_{\text {Win }}$ complex. MLL4 $W_{\text {in }}$ (in magenta) occupies the cavity of WDR5. On the right side, there is a cross-section schematic of the conical WDR5 cavity (in gray). MLL4 Win (magenta) partitions into the WDR5 cavity. This schematic is not on the same scale with the other cartoons.

produced current blockades showed two peaks, one at $\sim 60 \%$ and the other at $\sim 72 \%$. Plots of the normalized amplitude of WDR5produced current blockades as a function of WDR5-captured duration demonstrate that the short-lived events spanned the broadest range of $I / I_{0}$ (Supplementary Fig. 8). Earlier studies indicated that a $310^{\text {-helix is the bound conformation of MLL4 }} 4_{\text {Win }}$ to the Win binding site ${ }^{32}$. Here, we interpret that MLL4 $4_{\text {Win }}$ may also exhibit conformations that deviate from a 310 -helix. Such distinctive and more frequent conformers of a relatively flexible MLL4 $_{\text {Win }}$ likely generate the heterogeneity of its interactions with WDR5. The wide range of $I / I_{0}$ of the short-lived events likely correlates to many MLL4 $_{\text {Win }}$ conformers being able to bring about brief interactions. This interpretation also agrees with the medium- and long-lived events having closely similar $I / I_{0}$ values with a narrower range of normalized current amplitudes. Therefore, subtle alterations in current fluctuations between event types suggest that various conformers are present and the medium- and long-lived events are more selective as to what conformation $\mathrm{MLL}_{\text {Win }}$ must have to lead to a strong binding event.

Here, the association rate constants for short-, medium-, and long-lived events, $k_{\text {on-1 }}, k_{\text {on-2 }}$, and $k_{\text {on-3 }}$, respectively, were consistent for all [WDR5] values (Supplementary Table 7). Moreover, the frequency of short, medium-, and long-lived events was proportional to [WDR5] in a ratio 1:1 (Fig. 2h; Supplementary Fig. 9). This outcome indicates a bimolecular association process of the MLL4 $4_{\mathrm{Win}}$-WDR5 complex. The slopes of linear fits of the event frequency, $f\left(f=1 / \tau_{\text {on }}\right)$, versus [WDR5] were the corresponding $k_{\text {on }}$ values. Here, $k_{\text {on-1 }}, k_{\text {on-2 }}$, and $k_{\text {on-3 }}$ (mean \pm s.e.m.) were $(1.4 \pm 0.1) \times 10^{5} \mathrm{M}^{-1} \mathrm{~s}^{-1},(6.9 \pm 1.8) \times 10^{4} \mathrm{M}^{-1} \mathrm{~s}^{-1}$, and $(3.6 \pm 1.0) \times 10^{4} \mathrm{M}^{-1} \mathrm{~s}^{-1}$, respectively. Dissociation rate constants, $k_{\text {off-i }}(i=1,2$, and 3$)$, which were determined as reciprocal of the mean WDR5-captured durations $\left(1 / \tau_{\text {off-i }}\right)$, were independent of [WRD5] (Fig. 2i; Supplementary Table 8). This result suggests a unimolecular dissociation mechanism of the complex. Subscripts 1, 2, and 3 correspond to short-, medium-, and long-lived events, respectively. Fits of the dissociation rate constants versus [WDR5] resulted in their mean \pm s.e.m. values of $86 \pm 2 \mathrm{~s}^{-1}, 9.2 \pm 0.5 \mathrm{~s}^{-1}$, and $0.78 \pm 0.06 \mathrm{~s}^{-1}$, for short-, medium-, and long-lived WDR5 captures, respectively. The $K_{\mathrm{D}}$ for short-, medium-, and long-lived interactions were $631 \pm 49 \mu \mathrm{M}$, $138 \pm 18 \mu \mathrm{M}$, and $20 \pm 4 \mu \mathrm{M}$, respectively (Supplementary Table 9).

Biolayer interferometry validates the slow association process of the MLL4 $4_{\text {Win }}$-WDR5 complex. Then, we employed BLI, a real-time approach for determining the kinetics of $M L L 4_{\mathrm{Win}^{-}}$ WDR5 interactions in bulk phase (Methods). Here, we covalently attached MLL4 $4_{\text {Win }}$ onto the BLI sensor and recorded its binding interactions with WDR5. The association and dissociation phases were optically measured using changes in the interference pattern between reflected light waves at the sensor surface (Fig. 3a). The 


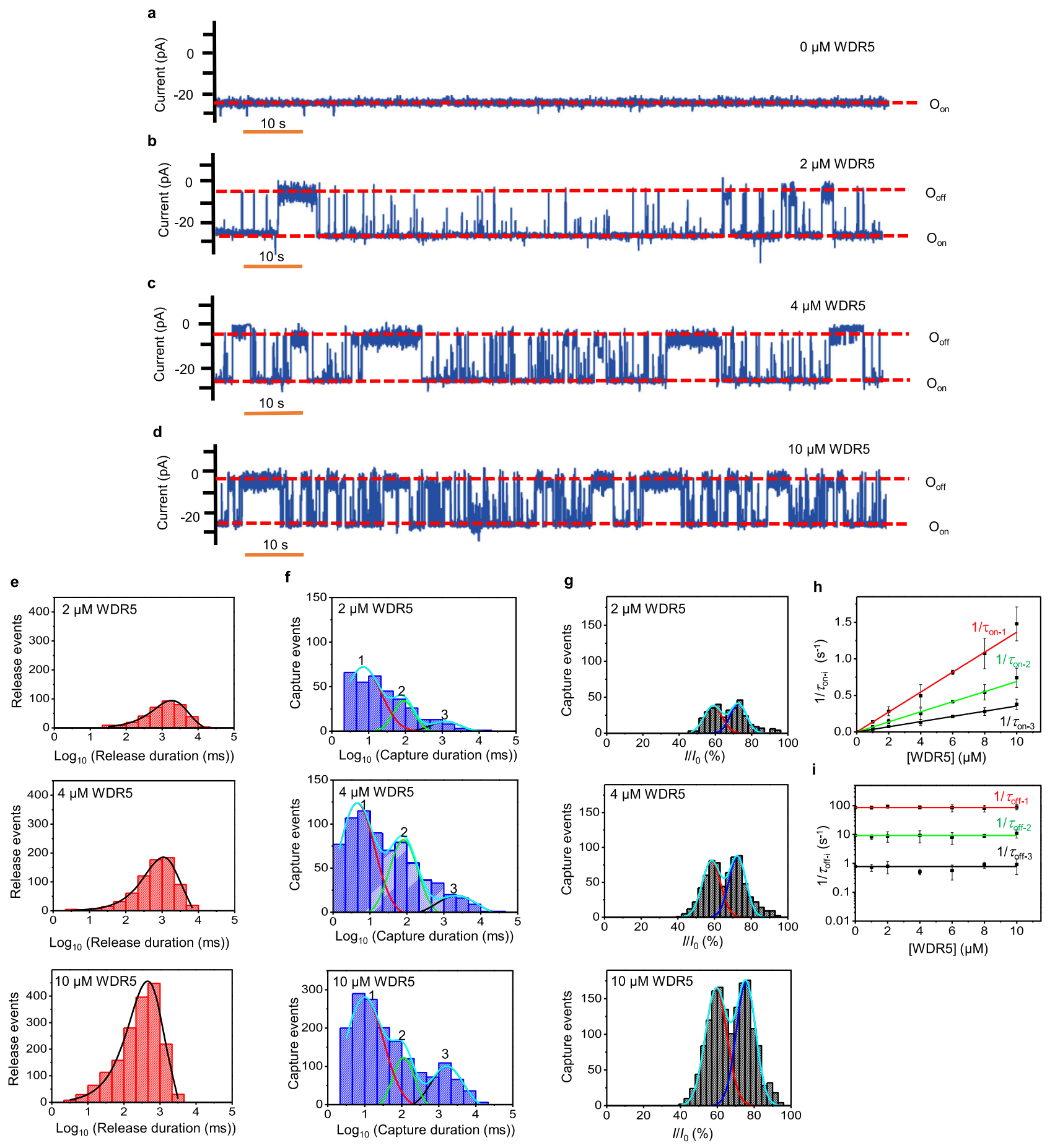

Fig. 2 Real-time detection of WDR5. a MLL4 ${ }_{\text {Win }}$ tFhuA. b MLL4 ${ }_{\text {Win }}$ tFhuA exposed to $2 \mu M$ WDR5. c MLL4 win tFhuA exposed to $4 \mu M$ WDR5. d MLL4 ${ }_{W i n}$ tFhuA exposed to $10 \mu M$ WDR5. $\mathrm{O}_{\text {on }}$ and $\mathrm{O}_{\text {off }}$ are release and capture substates, respectively. Data was replicated in three independent experiments. e Histograms of release durations, whose values (mean \pm s.e.m.) were $2.0 \pm 0.2 \mathrm{~s}$ (number of events: $N=363$ ), $1.2 \pm 0.4 \mathrm{~s}(\mathrm{~N}=717$ ), and $0.41 \pm 0.09 \mathrm{~s}(N=1791)$ at 2,4 , and $10 \mu \mathrm{M}$ WDR5, respectively. $\mathbf{f}$ Histograms of capture durations. The cumulative fits are marked in cyan. The red, green, and black curves indicate fits for short-, medium-, and long-lived captures, respectively. For $2 \mu \mathrm{M}$ WDR5, they (mean \pm s.e.m.) were $0.009 \pm 0.001 \mathrm{~s}$, $0.096 \pm 0.001 \mathrm{~s}$, and $1.4 \pm 0.1 \mathrm{~s}$, respectively (number of events: $N=329$ ). For $4 \mu \mathrm{M}$ WDR5, they were $0.008 \pm 0.001 \mathrm{~s}, 0.12 \pm 0.01 \mathrm{~s}$, and $2.0 \pm 0.1 \mathrm{~s}$, respectively $(N=717)$. For $10 \mu \mathrm{M}$ WDR5, they were $0.010 \pm 0.001 \mathrm{~s}, 0.10 \pm 0.01 \mathrm{~s}$, and $1.4 \pm 0.1 \mathrm{~s}$, respectively $(N=1624)$. $\mathrm{g}$ Histograms of normalized current blockades. The cumulative fits are marked in cyan. The red and blue curves indicate fits of smaller and larger blockades, respectively. For $2 \mu \mathrm{M}$ WDR5, these values (mean \pm s.e.m.) were $59 \pm 0.9 \%$ and $73 \pm 0.8 \%$, respectively (number of events: $N=329$ ). For $4 \mu \mathrm{M}$ WDR5, they were $58 \pm 0.8 \%$ and $73 \pm 0.7 \%$, respectively $(N=717)$. For $10 \mu \mathrm{M}$ WDR5, they were $59 \pm 0.6 \%$ and $75 \pm 0.1 \%$, respectively $(N=1607)$. $\mathbf{h}$ Dose response of $1 / \tau_{\text {on-i. }} . \tau_{\text {on- } 1}, \tau_{\text {on- }-2 \text {, }}$ and $\tau_{\text {on-3 } 3}$ are the mean durations between short-, medium-, and long-lived captures, respectively. $\mathbf{i}$ Dose response of $1 / \tau_{\text {off-i }}$. $\tau_{\text {off- }-1}, \tau_{\text {off-2, }}$, and $\tau_{\text {off-3 } 3}$ are the mean durations of short-, medium-, and long-lived captures, respectively. $\ln (\mathbf{h})$ and (i), data represent mean \pm s.d. obtained from either $n=4(1$ and $2 \mu M$ WDR5) or $n=3(4,6,8$, and $10 \mu$ M WDR5) distinct experiments. Source data are provided as a Source Data file. 
a
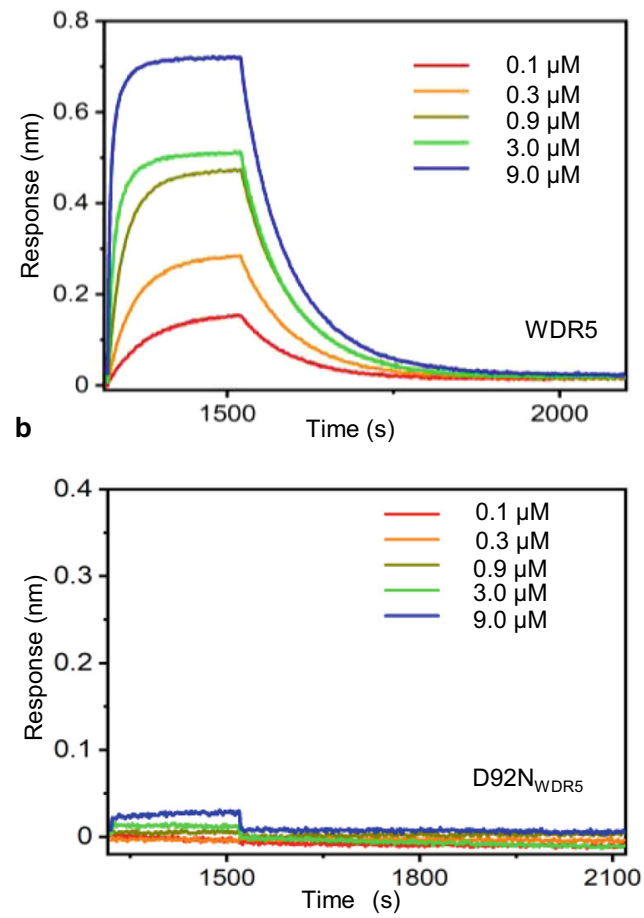

Fig. 3 Biolayer interferometry (BLI) sensorgrams of MLL4 $_{\mathbf{W i n}}-$ WDR5 interactions. a $5 \mathrm{nM}$ biotin-tagged $M L L 4_{\text {Win }}$ peptide was loaded onto streptavidin sensors for $5 \mathrm{~min}$. Individual binding curves are indicated for [WDR5] from $0.1 \mu \mathrm{M}$ to $9 \mu \mathrm{M}$. b The same BLI measurements as described in (a), but conducted with D92N WDR5. Source data are provided as a Source Data file.

BLI-measured $k_{\text {on }}$ was $(2.6 \pm 0.1) \times 10^{4} \mathrm{M}^{-1} \mathrm{~s}^{-1}$ (Supplementary Table 10). The BLI-measured $k_{\text {off }}$ was $(1.2 \pm 0.1) \times 10^{-2} \mathrm{~s}^{-1}$, to generate a $K_{\mathrm{D}}$ of $(0.46 \pm 0.04) \times 10^{-6} \mathrm{M}$. BLI was unable to resolve the three binding subpopulations observed when using $\mathrm{MLL}_{\mathrm{Win}} \mathrm{tFhuA}$ and only yielded one $k_{\text {on }}$ and one $k_{\text {off }}$. We also employed BLI to conduct a positive-control experiment, testing whether our peptide adaptor exhibits any interaction with WDR5 and found none (Supplementary Fig. 10).

To better understand the mechanistic distinctions of the nanopore and BLI measurements, we simulated the BLI response by using results from single-channel electrical recordings. The simulation shows that the majority of the contribution to the observed BLI response is generated by the long-lived MLL4 $4_{\mathrm{Win}}$-WDR5 interactions (Supplementary Fig. 11). This confirms our tentative interpretation that the BLI sensorgram is extensively biased by the impact of the long-lived binding interactions, despite their lowest event frequency. This simulated curve highlights the superiority of single-molecule measurements using our protein nanopore due to their broad time bandwidth.

The three binding events involve Win binding site interactions. Next, we employed D92 $\mathrm{N}_{\text {WDR5 }}$, a WDR5 variant, to find out which event types are related to the partitioning of MLL4 ${ }_{\text {Win }}$ into the cavity. Also, the detection of $\mathrm{D} 92 \mathrm{~N}_{\text {WDR5 }}$ provided proof that our MLL $_{\text {win }}$ tFhuA can recognize a somatic cancer mutant of WDR $5^{40}$. Asp-92 is a residue deeply located at the inner tip of the WDR5 cavity (Fig. 1b; Supplementary Fig. 12). First, we used BLI to evaluate MLL4 $4_{\mathrm{Win}}-\mathrm{D} 92 \mathrm{~N}_{\mathrm{WDR} 5}$ interactions. Interestingly, MLL4 $4_{\text {Win }}$ binds very weakly to D92 $\mathrm{N}_{\mathrm{WDR} 5}$, and we were unable to quantify these interactions using BLI (Fig. 3b). D92 $\mathrm{N}_{\mathrm{WDR}}$ produced current blockades with an amplitude comparable to that of
WDR5-captured events (Fig. 4a, b). Moreover, D92N $\mathrm{N}_{\text {WDR5-cap- }}$ tured events appeared in a concentration-dependent manner (Fig. 4b-d). As in the WDR5 case, we noted a broad span of

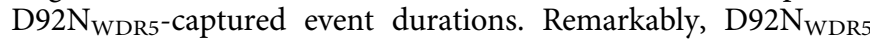
also showed three distinct binding events, as judged by the LLR test (Fig. 4e, f; Supplementary Table 11). However, the frequency of D92 $\mathrm{N}_{\text {WDR5-captured events was drastically reduced with }}$ respect to that value of the WDR5-captured events. This result indicates the influential role of Asp-92 in MLL4 $4_{W i n}-W D R 5$ interactions. In addition, the three events had D92 $\mathrm{N}_{\mathrm{WDR} 5}$-captured durations similar to those recorded with WDR5. Therefore, the conservation of the three-component binding events illuminates that these subpopulations resulted from interactions deeply located within the WDR5 cavity. Event probabilities of short-, medium-, and long-lived D92 $\mathrm{N}_{\text {WDR5-captured events also fol- }}$ lowed the inequality: $P_{1}>P_{2}>P_{3}$ (Supplementary Table 12). Again, it is likely that these three interactions correspond to three different modes of $\mathrm{D} 92 \mathrm{~N}_{\mathrm{WDR}}$ recognition by the flexible $\mathrm{MLL}_{\mathrm{Win}}$ ligand ${ }^{41}$. The similarity between $\mathrm{D} 92 \mathrm{~N}_{\mathrm{WDR} 5}$ and WDR5 shows that these different MLL4 $_{W i n}$ conformers were present in the interactions of MLL4 $4_{\text {Win }}$ with each protein. This outcome confirms closely related multimodal binding mechanisms of the two proteins. The D92N mutation influences the $k_{\mathrm{on}}$, but not the $k_{\text {off }}$ of short-, medium-, and long-lived D92 $\mathrm{N}_{\mathrm{WDR} 5^{-}}$ captured events (Supplementary Table 13). Furthermore, the frequency of each of the three event types depended on D92 $\mathrm{N}_{\text {WDR5 }}$ concentration, [D92 $\mathrm{N}_{\mathrm{WDR} 5}$ ], in a 1:1 ratio (Supplementary Figs. 13, 14 Table 14). Hence, our findings suggest that the three events were specific binding events. $K_{\mathrm{D}}$ values corresponded to about one order of magnitude weaker interactions than those obtained with WDR5, regardless of event type. For short-, medium-, and long-lived $\mathrm{D} 92 \mathrm{~N}_{\mathrm{WDR} 5}$-produced interactions, $K_{\mathrm{D}}$ values were $5.4 \pm 1.3 \mathrm{mM}, \quad 721 \pm 18 \mu \mathrm{M}$, and $218 \pm 22 \mu \mathrm{M}$, respectively (Supplementary Table 15). This result reveals that our protein nanopore is able to probe extremely weak interactions in the low millimolar range. Our approach also provides quantitative distinctions between the binding interactions of D92 $\mathrm{N}_{\text {WDR5 }}$ and WDR5 (Supplementary Tables 16,17).

Fits of individual rate constants, which are presented above, were conducted with the assumption that no transitions occur among the three capture substates. However, it is not clear whether a kinetic model encompassing interconversion transitions among these capture substates would be better suited for experimentally determined rate constants. An interconversiondependent kinetic model was created, including six additional rate constants among the capture substates (Supplementary Tables 18, 19 Figs. 15-16). At a confidence level $C>0.95$, we found that fits to an interconversion-dependent kinetic model were not statistically better than those corresponding to an interconversion-independent kinetic model, as judged by the LLR test.

A negative charge removal on the WDR5 local surface does not impact the WDR5-MLL4 ${ }_{\text {Win }}$ interaction. The weak interaction between D92 $\mathrm{N}_{\mathrm{WDR} 5}$ and MLL4 $4_{\mathrm{Win}}$ prompted the examination of D172A $\mathrm{A}_{\text {WDR5 }}$ (Supplementary Fig. 17), a second WDR5 mutant, which served for an additional positive-control experiment. Here, the goal was to observe how a small alteration in the local surface charge of WDR5 influences the observed kinetic fingerprint of its interactions with MLL4 $4_{\text {Win }}$. The removal of an Asp residue on the protein surface was critical to have a reliable comparison with D92 $\mathrm{N}_{\text {WDR5 }}$. D172 $\mathrm{A}_{\text {WDR5 }}$ was selected, because it maintains the integrity of the WDR5 cavity while altering the local surface charge of the protein in the proximity of the Win binding site. We found that $\mathrm{D}_{172 \mathrm{~A}_{\mathrm{WDR}} \text { produced current blockades with release }}$ 
a

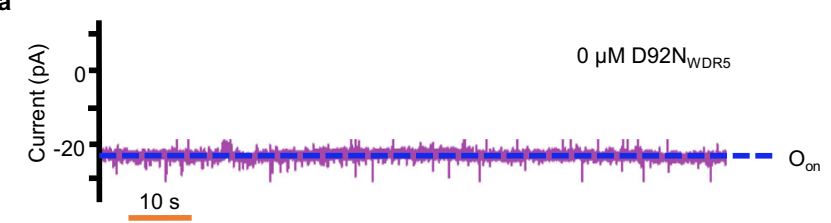

b

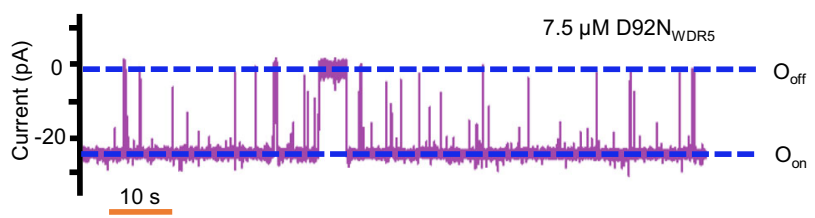

d
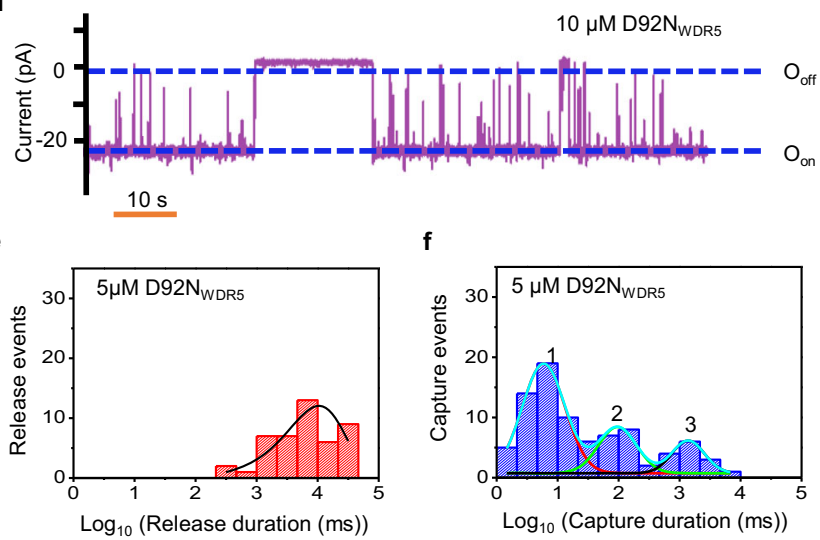

f
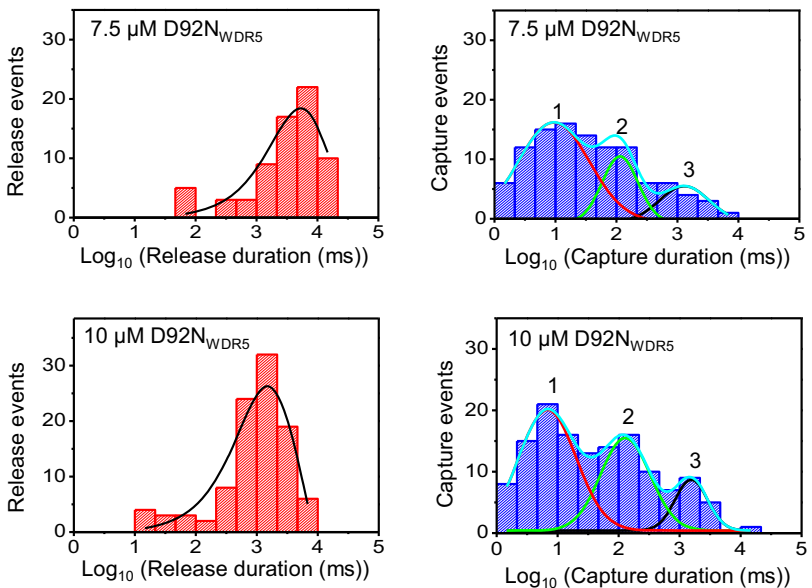

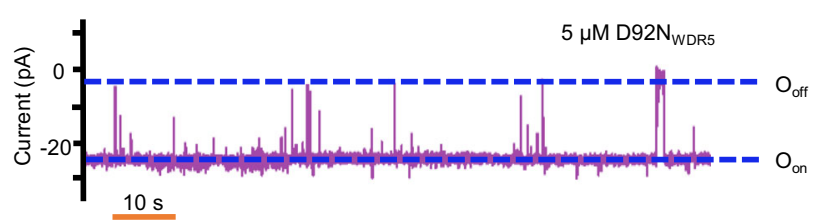

Fig. 4 Real-time detection of weakly binding $D_{92} N_{\text {WDR5. }}$ a $A$ singlechannel electrical trace of MLL4 ${ }_{\text {Win }}$ tFhuA. b MLL4 ${ }_{W_{i n}}$ tFhuA with $5 \mu \mathrm{M}$

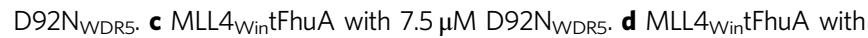
$10 \mu \mathrm{M}$ D92 $\mathrm{N}_{\text {WDR5. }} \mathrm{O}_{\text {on }}$ and $\mathrm{O}_{\text {off }}$ are release and capture substates, respectively. This single-channel electrical signature was replicated in three independent experiments. Single-channel electrical traces were low-pass filtered at a frequency of $100 \mathrm{~Hz}$ using an 8-pole Bessel filter. e Histograms of D92 $\mathrm{N}_{\text {WDR5 }^{-}}$ released durations $\left(\tau_{\text {on }}\right)$ at $5 \mu \mathrm{M}, 7.5 \mu \mathrm{M}$, and $10 \mu \mathrm{M}$ D92 $\mathrm{N}_{\text {WDR5. }} \tau_{\text {on }}$ durations (mean \pm s.e.m.) were $9.1 \pm 3.4 \mathrm{~s}$ (number of events: $N=45$ ), $4.9 \pm 1.0 \mathrm{~s}$ $(N=69)$, and $2.2 \pm 0.8 \mathrm{~s}(N=101)$, respectively. $\mathbf{f}$ Histograms of $D 92 \mathrm{~N}_{\text {WDR5 }^{-}}$ captured durations $\left(\tau_{\text {off }}\right)$. The cumulative fits are marked in cyan. The red, green, and black curves indicate fits of $\tau_{\text {off-1 }}, \tau_{\text {off- } 2 \text {, }}$ and $\tau_{\text {off-3 }}$ for short-, medium-, and long-lived D92 $\mathrm{N}_{\text {WDR5 }}$-captured events, respectively. For $5 \mu \mathrm{M}$ D92 $\mathrm{N}_{\text {WDR5 }}$ they (mean \pm s.e.m.) were $0.008 \pm 0.001 \mathrm{~s}, 0.13 \pm 0.01 \mathrm{~s}$, and $1.6 \pm 0.1 \mathrm{~s}$, respectively (number of events: $N=78$ ). For $7.5 \mu \mathrm{M}$ D92 $\mathrm{N}_{\mathrm{WDR} 5}$, they were $0.010 \pm 0.001 \mathrm{~s}, 0.12 \pm 0.01 \mathrm{~s}$, and $1.9 \pm 0.1 \mathrm{~s}$, respectively $(N=106)$. For $10 \mu \mathrm{M}$ D92N $N_{\text {WDR5, }}$ they were $0.009 \pm 0.001 \mathrm{~s}, 0.19 \pm 0.01 \mathrm{~s}$, and $1.8 \pm 0.1 \mathrm{~s}$, respectively $(N=120)$. Data was extracted from 20 min recordings. Source data are provided as a Source Data file.

similar binding events to those described above (Supplementary Figs. 20, 21). The frequencies of all three WDR5-captured events depended on [WDR5], in a 1:1 ratio, whereas their durations were independent of [WDR5] (Supplementary Fig. 22 and Tables 26-30). This finding suggests that the three observed events were specific binding modes between one tethered $\mathrm{MLL}_{\text {win }}$ ligand and one WDR5 protein. The unaltered $k_{\text {off-i }}$ under this condition with respect to values obtained at a transmembrane potential of $-20 \mathrm{mV}$ shows that the integrity of the deep binding interface of WDR5 is not influenced by the transmembrane potential.

Voltage dependence of MLL4 ${ }_{\text {Win }}$-WDR5 interactions. WDR5 has a slightly positive charge under our experimental conditions and binding interactions occur near the nanopore opening. Therefore, we hypothesized that the $k_{\text {on-i }}$ is voltage dependent, so we compared single-channel electrical traces at various transmembrane potentials when $8 \mu \mathrm{M}$ [WDR5] was kept unchanged in the cis compartment. In accord with our expectation, the three WDR5 capture rate constants, $k_{\text {on-i }}(i=1,2$, and 3$)$, increased at elevated negative transmembrane potentials (Fig. 5a-d and Supplementary Figs. 23, 24, Table 31). Our detailed event analyses also confirmed that the $k_{\text {off-i }}(i=1,2$, and 3$)$ and distribution probabilities of the three protein recognition events were independent of the transmembrane potential (Fig. $5 \mathrm{e}$ and Supplementary Tables 32, 33). In addition, a semilogarithmic representation of the voltage dependence of all $k_{\text {on-i }}$ values revealed an identical slope for the three binding events, suggesting a similar mechanism of these WDR5 recognition modes. Hence, this enabled the calculation of the association rate constants at a zero transmembrane potential for the three binding events, $k_{\text {on-i }}(0)$ (Supplementary Table 34). For example, the lower-limit association constant of the long-lived events at a zero transmembrane potential, $k_{\text {on-3 }}(0)$, was $(1.9 \pm 0.2) \times 10^{4} \mathrm{M}^{-1} \mathrm{~s}^{-1}$, which compares well with the BLIdetermined $k_{\text {on }}$ under similar buffer conditions (Supplementary Table 10). The reduction in the activation free energy of all WDR5released events, $\Delta \Delta G_{\text {on }}$, was $\sim 0.7 \mathrm{kcal} / \mathrm{mol}$ at a transmembrane potential of $-40 \mathrm{mV}$ with respect to that value determined at a zero transmembrane potential (Supplementary Table 35). This data was used to determine a small relative net charge, $z$, of $\sim 0.8$ for WDR5 (Supplementary Table 36).

The three protein recognition modes are conserved at a higher transmembrane potential. We then explored whether this kinetic fingerprint of MLL4 $4_{\mathrm{Win}}-\mathrm{WDR} 5$ interactions exists under different experimental conditions. Therefore, we first conducted singlemolecule experiments at a transmembrane potential of $-40 \mathrm{mV}$. The addition of WDR5 to the cis compartment produced closely
Quantitative and conceptual comparisons of nanopore sensing with BLI. In a recent study ${ }^{42}$, we have shown that the $k_{\text {on }}$ values of Win motif peptides with WDR5 are influenced by their 
tethering on a sensor surface. In addition, the partitioning of MLL4 $_{\text {Win }}$ into the WDR5 cavity significantly reduces the $k_{\text {on }}$ due to the entropic penalty of this process ${ }^{32}$. The nanoporedetermined $k_{\text {on }}$ ranged between $10^{4}$ and low $10^{5} \mathrm{M}^{-1} \mathrm{~s}^{-1}$. These values and the BLI-determined $k_{\text {on }}$ value are at least two orders of magnitude lower than one would expect for the $k_{\text {on }}$ of peptideprotein complexes $\left(10^{7}-10^{8} \mathrm{M}^{-1} \mathrm{~s}^{-1}\right)^{43,44}$. Interestingly, the BLIdetermined $k_{\text {on }}$ was similar to $k_{\text {on-3 }}$ probed by nanopore recordings. This finding suggests that the similar tethering and entropic penalty in both techniques influence the $k_{\text {on }}$. On the other hand, the BLI-determined $k_{\text {off }}$ was much lower than all nanopore-inferred $k_{\text {off-i }}$ values. Yet, it must be noted that the inability of BLI to distinguish the three binding events and its relatively limited time resolution make it challenging to compare dissociation rate constants. Instead, our approach can be employed to detect a significantly weaker interaction than that probed by BLI. This protein nanopore is the only tool sensitive enough to resolve the three modes of protein recognition in real time.

Distinctive outcomes with WDR5 and D92 $\mathrm{N}_{\text {WDR5. In this work, }}$ we developed a modular protein nanostructure capable of discriminating between three individual kinetic components existing in a complex binding distribution (Fig. 6a). Furthermore, a weakly binding mutant, $\mathrm{D} 92 \mathrm{~N}_{\mathrm{WDR} 5}$, confirmed the three-binding event distribution. D92 $\mathrm{N}_{\mathrm{WDR} 5}$ does not show an altered $k_{\text {off }}$, because Asp-92 does not form any stabilizing bonds with MLL4 $_{\text {Win }}{ }^{32,33}$. Only Cys-261, Phe-133, Ser-91, and Asp-107 are responsible for the stability of this interaction (Supplementary Fig. 1 Table 2). Therefore, both proteins show a similar activation free energy of dissociation, $\Delta G_{\text {off }}$ (Fig. 6b). However, Asp-92 of WDR5 does provide a negative charge to assist with the electrostatic pulling of the critical Arg residue of MLL4 $4_{\text {Win }}$ into the WDR5 cavity (Supplementary Table 1 Fig. 12) ${ }^{45}$. In contrast, Asn-92 of D92 $\mathrm{N}_{\mathrm{WDR} 5}$ does not exert this role, so the activation free energy barrier of association, $\Delta G_{\text {on }}$, is significantly amplified, reducing the frequency of reversible binding events.

Advantages of this protein nanopore. Our nanopore experiments with WDR5, D92 $\mathrm{N}_{\mathrm{WDR}}$, and $\mathrm{D} 172 \mathrm{~A}_{\mathrm{WDR} 5}$, along with prior crystallographic studies ${ }^{22,33}$, suggest that all three binding events are specific interactions and occur deeply within the WDR5 cavity. In addition, both D92 $\mathrm{N}_{\mathrm{WDR} 5}$ and D172 $\mathrm{A}_{\text {WDR5 }}$ showed normalized current blockades and capture durations similar to those noted with WDR5 (Fig. 2g; Supplementary Figs. 8 and 25, Tables 4, 6, 11,20,22, and 37). Through this cascade of binding scenarios, we show that our protein nanopore is capable of sampling complex binding interfaces. It could also be used to study other WDRs and groove-containing binding systems. These include unfolded protein chains entering chaperone tunnels, such as those of the GroEL ${ }^{46}$ and $\mathrm{Clp}^{47}$ families. Furthermore, we show that our protein nanopore can also probe very weak interactions with affinities up to low micromolar. Again, this substantially extends the application spectrum of our nanopore and highlights its significant sensitivity. For example, the interaction details observed in our study could only be observed at single-molecule precision. Other bulk-phase techniques, such as isothermal titration calorimetry (ITC ${ }^{48}$ and surface plasmon resonance $(\mathrm{SPR})^{49}$, can be used to study these peptide-protein interactions, but they have a narrower time bandwidth. These limitations of ensemble studies prevent us from identifying binding details like those present in a realistic biological system.

Implications and prospects in biotechnology. Recently, it has been discovered that the high-affinity Win binding site of WDR5 is a key player in transient interactions with dozens of proteins $s^{50}$. These include 3-phosphoinositide-dependent protein kinase 1 (PDPK1) and proteins involved in phosphatidylinositol 3-kinase (PI3K) signaling. Here, we speculate that WDR5 selectively utilizes the Win binding site. For example, it is likely that the long-lived interactions of WDR5 have physiological relevance for the functional integrity of large multi-subunit complexes of methyltransferases ${ }^{51}$, which is required for optimal enzymatic activity ${ }^{52-54}$. On the other hand, weaker interactions of WDR5 with other proteins may assist in cell signaling pathways ${ }^{50}$. Hence, the wide range of binding affinities of these specific interactions is in accord with the multitasking feature of the Win binding site in subnuclear PPIs ${ }^{55,56}$. Furthermore, there is significant interest in exploring the Win binding site, because WDR5 is implicated in numerous cancers ${ }^{57,58}$. Therefore, our protein nanopore can serve as a tool to acquire real-time and comprehensive pharmacokinetics for these clinically important interactions.

In summary, we probed the complexity of MLL4 $4_{\mathrm{Win}}$-WDR5 interactions with no steric hindrance of nanopore confinement. The heterogeneous event distribution unambiguously revealed three distant modes of protein recognition with diverse affinities. The interactions of MLL4 Win $_{\text {in }}$ with a weakly binding WDR5 derivative were not quantifiable using BLI, but our nanopore sensor provided a complete characterization of the binding kinetics. This unusual kinetic fingerprint of MLL4 $4_{\mathrm{Win}}-\mathrm{WDR} 5$ interactions was further confirmed and fully characterized at varying voltage biases. Therefore, this study demonstrates that our approach can illuminate a wide span of multimodal protein recognition events and strengthen quantitative protein interaction studies.

\section{Methods \\ Modular genetic engineering of the protein nanopore. A plasmid with} omll4tfhua as the gene of interest was purchased from GenScript (Piscataway, NJ). This gene included a DNA sequence encoding, from the $\mathrm{N}$ to $\mathrm{C}$ terminus, a 13residue peptide adaptor (MGDRGPEFELGTM), a 14-residue MLL4 Win motif peptide (MLL4 Win $_{\text {in }}$ LNPHGAARAEVYLR), a 6-residue Gly/Ser-rich peptide tether, and a 455-residue large truncation of Ferric hydroxamate uptake component A of Escherichia coli (tFhuA) ${ }^{12}$. The pPR-IBAl vector was used as the template. The MLL4 $4_{\text {Win }}$ represented a recognition element for target analytes WDR5, D92N WDR5 $_{\text {, }}$ and $\mathrm{D} 172 \mathrm{~A}_{\mathrm{WDR} 5}$. The peptide adaptor was slightly negatively charged and unstructured in solution ${ }^{59}$. This 488 -residue modular nanopore was named MLL4 $4_{\text {Win }}$ tFhuA.

Protein expression and purification. The plasmid mentioned above was transformed into E. coli BL21(DE3) cells ${ }^{13,34}$. All transformed cells were grown in a Luria-Bertani medium at $37^{\circ} \mathrm{C}$ until the $\mathrm{OD}_{600}$ reached a value of $\sim 0.4$. Then the cells were induced using $1 \mathrm{mM}$ isopropyl $\beta$-D-1-thiogalactopyranoside (IPTG) and left for further culturing for $\sim 5 \mathrm{~h}$ at $37^{\circ} \mathrm{C}$. Next, the cells were centrifuged at $3700 \times g$ for $30 \mathrm{~min}$ at $4^{\circ} \mathrm{C}$, then resuspended in $300 \mathrm{mM} \mathrm{KCl}, 20 \mathrm{mM}$ Tris- $\mathrm{HCl}$, $5 \mathrm{mM}$ ethylenediaminetetraacetic acid (EDTA), $\mathrm{pH}$ 8. Cell lysis was achieved using a microfluidizer (Model $110 \mathrm{~L}$; Microfluidics, Newton, MA). The cell lysate was centrifuged at $108,500 \times g$ for $30 \mathrm{~min}$ at $4{ }^{\circ} \mathrm{C}$ to separate the insoluble pellet and supernatant. The supernatant was discarded, and the water-insoluble modular protein remained in the pellet. The pellet encapsulated the protein inclusion bodies and went through a series of $1.5 \%$ Triton X-100, $1 \mathrm{mM}$ EDTA washes in order to remove cellular debris. The supernatant was centrifuged at $108,500 \times g$ for $30 \mathrm{~min}$ at $4{ }^{\circ} \mathrm{C}$ to separate target insoluble protein from the water-soluble cellular debris. The precipitate was solubilized in $8 \mathrm{M}$ urea. Then, the solubilized protein was further purified on an anion-exchange column (Q12-Sepharose; Bio-Rad, Hercules, CA) using a linear salt gradient of $0-1 \mathrm{M} \mathrm{KCl}, 20 \mathrm{mM}$ Tris- $\mathrm{HCl}, \mathrm{pH}$. The peak fractions were collected, combined, and centrifuged at $3700 \times g$ for $10 \mathrm{~min}$ at $4{ }^{\circ} \mathrm{C}$ to separate precipitated proteins. These fractions were passed through a sizeexclusion column (HiLoad16/600 Superdex-75; GE Healthcare Life Sciences,

Pittsburg, PA) for a final purification step. The fractions correlating to the target protein size were combined and centrifuged $3700 \times g$ for $10 \mathrm{~min}$ at $4{ }^{\circ} \mathrm{C}$ to remove aggregated protein. The supernatant was removed and prepped for lyophilization. The protein purity was assessed using SDS-PAGE analyses.

WDR5, D92 $\mathrm{N}_{\text {WDR5 }}$, and D172A $\mathrm{A}_{\text {WDR5 }}$ were expressed using Rosetta II pLysS (Novagen via Millipore Sigma, Burlington, MA) competent cells ${ }^{30,60}$. All transformed cells were grown in Luria-Bertani medium at $37^{\circ} \mathrm{C}$ until the $\mathrm{OD}_{600}$ reached 0.75 and cells were then chilled at $4{ }^{\circ} \mathrm{C}$ until they reached an $\mathrm{OD}_{600}$ value of 1.0. The cells were induced with $1 \mathrm{mM}$ IPTG and left for further growth for $18-20 \mathrm{~h}$ at $16^{\circ} \mathrm{C}$. Cells were centrifuged at $4000 \times g$ for $30 \mathrm{~min}$ at $4{ }^{\circ} \mathrm{C}$ to harvest the 
a

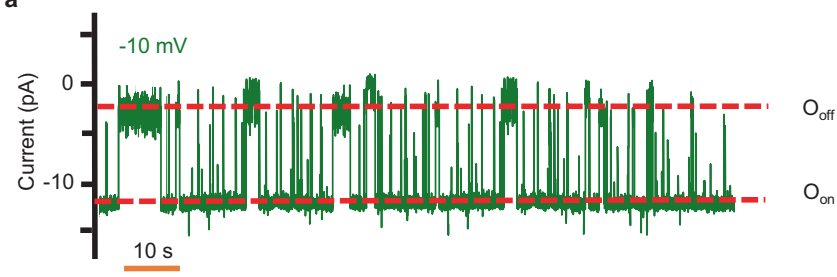

b
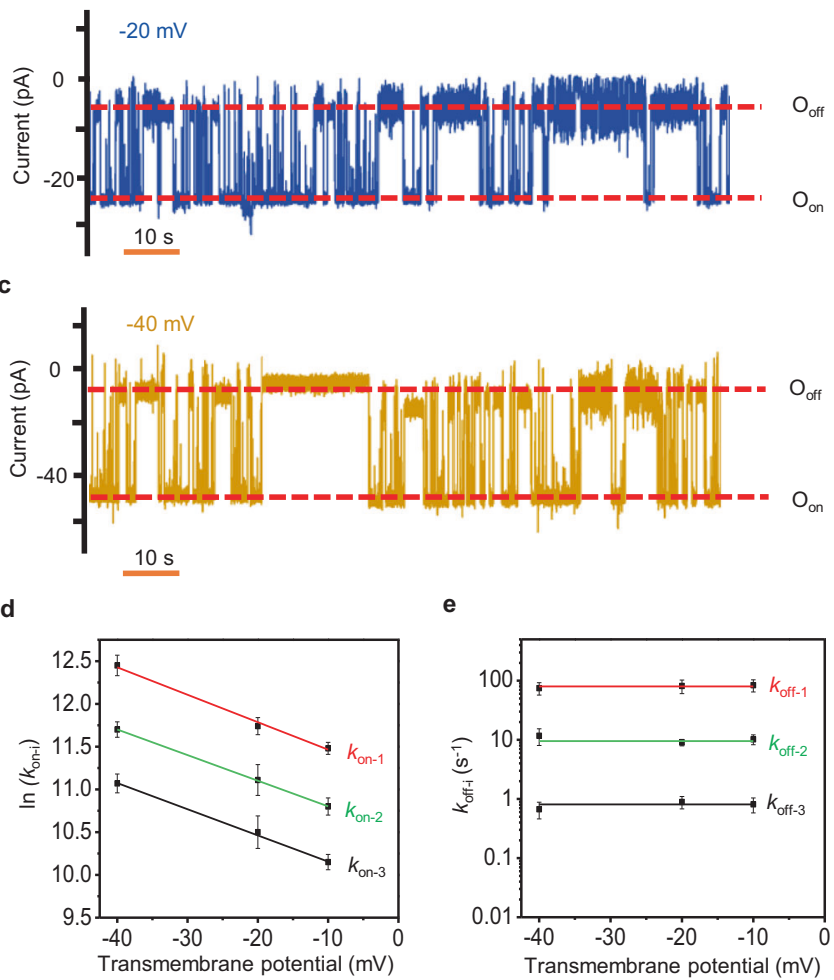

Fig. 5 Voltage dependence of MLL4 $_{\text {Win }}$-WDR5 interactions. a Reversible WDR5 captures were observed through current transitions between the $\mathrm{O}_{\text {on }}$ and $\mathrm{O}_{\text {off }}$ substates of MLL4 ${ }_{\text {win }}$ tFhuA at an applied transmembrane potential of $-10 \mathrm{mV}$. b The same as in (a), but at an applied transmembrane potential of $-20 \mathrm{mV}$. c The same as in (a), but at an applied transmembrane potential of $-40 \mathrm{mV} . \mathrm{O}_{\text {on }}$ and $\mathrm{O}_{\text {off }}$ are release and capture substates, respectively. This single-channel electrical signature was replicated in three independent experiments. Single-channel electrical traces in (a)-(c) were low-pass filtered at $100 \mathrm{~Hz}$ using an 8-pole Bessel filter. d Linear plot in a semilogarithmic representation, which shows the dependence of $\ln \left(k_{\text {on-i }}\right)$ on the transmembrane potential, where $i=1,2$, and 3 . Here, $k_{\text {on-i, }}$ with $i=1,2$, and 3, are the association rate constants of short-, medium, and long-lived binding events, respectively. e The dependence of $k_{\text {off-i }}$ on the transmembrane potential. Here, $k_{\text {off-i }}$, with $i=1,2$, and 3 , are the reciprocal of mean durations of short-, medium-, and long-lived WDR5 captures, respectively. In (d) and (e), data points represent mean \pm s.d. obtained from $n=3$ distinct experiments. $8 \mu \mathrm{M}$ [WDR5] was added to the cis compartment. Source data are provided as a Source Data file.

pellet. The pellet was resuspended in $300 \mathrm{mM} \mathrm{KCl,} 50 \mathrm{mM}$ Tris, $\mathrm{pH}$ 7.4, $3 \mathrm{mM}$ dithiothreitol (DTT), $30 \mathrm{mM}$ imidazole, $0.1 \mathrm{mM}$ phenylmethylsulfonyl fluoride (PMSF), and one EDTA-free protease inhibitor cocktail tablet (cOmplete; Sigma Aldrich, St. Louis, MO). Cell lysis was achieved using a microfluidizer (Model M110L; Microfluidics, Newton, MA). The lysate was cleared by centrifuging at $108,500 \times g$ at $4{ }^{\circ} \mathrm{C}$ for 35 minutes. The supernatant containing WDR5, D92 $\mathrm{N}_{\text {WDR5 }}$ or D172A $\mathrm{A}_{\text {WDR5 }}$ was passed through an immobilized metal-affinity column $(5 \mathrm{~mL}$, Bio-Scale Mini Profinity IMAC cartridge; Bio-Rad, Hercules, CA). The protein was then eluted with a 20 -column volume linear gradient of imidazole until the final buffer was $300 \mathrm{mM} \mathrm{KCl,} 50 \mathrm{mM}$ Tris- $\mathrm{HCl}, \mathrm{pH}$ 7.4, $3 \mathrm{mM}$ DTT, and $500 \mathrm{mM}$ imidazole. Then an SDS-PAGE gel was run to determine which fractions to collect

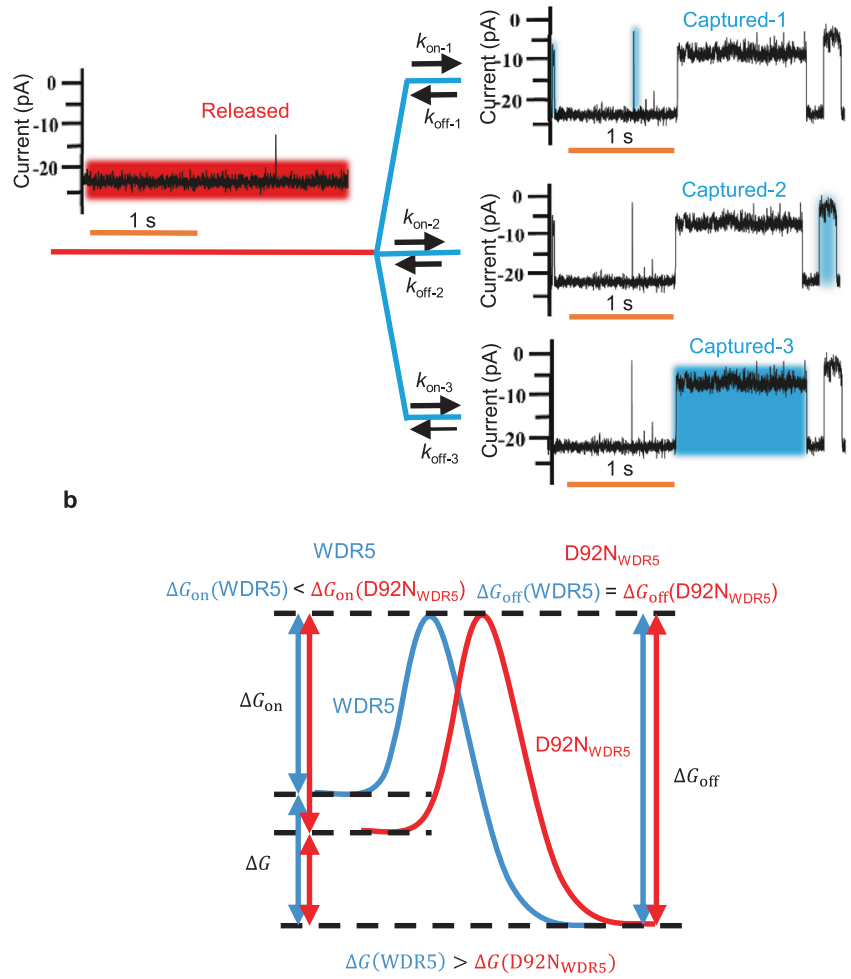

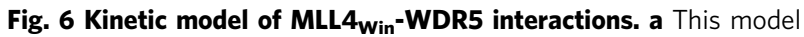
includes the open current substate, $\mathrm{O}_{\text {on }}$ (highlighted in red), and the three protein recognition events, $\mathrm{O}_{\text {off }}$ (highlighted in blue). $\mathbf{b}$ Model of the free energy landscape of MLL4 ${ }_{W i n}-$ WDR5 interactions for both WDR5 and D92NWDR5.

for further purification. Tobacco Etch Virus (TEV) protease (New England Biolabs, Ipswich, MA) was used to remove the hexahistidine tag on the protein. After TEV digestion, the protein sample was dialyzed against $300 \mathrm{mM} \mathrm{KCl}, 50 \mathrm{mM}$ Tris, $\mathrm{pH}$ 7.4, $3 \mathrm{mM}$ DTT and $30 \mathrm{mM}$ imidazole, so it could be passed over the immobilized metal-affinity column (5 mL, Bio-Scale Mini Profinity IMAC cartridge; Bio-Rad) for a second run. Finally, protein samples were concentrated using a $10 \mathrm{kDa}-$ molecular weight cut-off spin concentrator (Millipore Sigma, St. Louis, MO). The concentrated samples went through a final gel-filtration purification process using a size-exclusion column (HiLoad16/600 Superdex-75;GE Healthcare Life Sciences, Pittsburg, PA). The final sample buffer was $300 \mathrm{mM} \mathrm{KCl}, 50 \mathrm{mM}$ Tris, $1 \mathrm{mM}$ tris (2carboxyethyl)phosphine (TCEP), pH 7.4. The concentrated protein sample was run on an SDS-PAGE gel to confirm size and purity.

Protein refolding. MLL4 ${ }_{\mathrm{Win}} \mathrm{tFhuA}$ was solubilized from a lyophilized form in $200 \mathrm{mM} \mathrm{KCl}, 8 \mathrm{M}$ urea, $20 \mathrm{mM}$ Tris- $\mathrm{HCl}, \mathrm{pH} 8$ to a concentration of $\sim 25 \mu \mathrm{M}$ and incubated at room temperature for at least one hour. After protein quantification via molar absorptivity, $n$-dodecyl- $\beta$-D-maltopyranoside (DDM; Anatrace, Maumee, $\mathrm{OH})$ was added to the denatured samples to a final concentration of $1 \%(\mathrm{w} / \mathrm{v})$. The solubilized protein was placed in a dialysis bag with a $14 \mathrm{kDa}$ molecular weight cut off, then dialyzed against the optimized refolding buffer, which was $200 \mathrm{mM}$ $\mathrm{KCl}, 20 \mathrm{mM}$ Tris- $\mathrm{HCl}, \mathrm{pH} 8$, at $4{ }^{\circ} \mathrm{C}$ for at least $72 \mathrm{~h}$. The dialysis solution was replaced once every $24 \mathrm{~h}$. This refolded protein sample was then centrifuged to eliminate any precipitation of unfolded proteins. The supernatant was separated as the working sample for single-channel electrical recordings. Protein quantification was determined using molar absorptivity at a wavelength of $280 \mathrm{~nm}$.

Biolayer interferometry. These experiments were executed using an Octet Red384 instrument (FortéBio, Fremont, CA $)^{42}$. MLL4 $4_{\text {Win }}$ was biotinylated and amidated at the $\mathrm{N}$ and $\mathrm{C}$ terminus, respectively. A buffer solution containing $300 \mathrm{mM} \mathrm{KCl}$, $20 \mathrm{mM}$ Tris- $\mathrm{HCl}, 1 \mathrm{mM}$ TCEP, $1 \mathrm{mg} / \mathrm{ml}$ bovine serum albumin (BSA), pH 7.5 was used to soak streptavidin (SA) sensors for $30 \mathrm{~min}$. A flexible (GGS) ${ }_{3}$ linker was inserted between the MLL4 $4_{\text {Win }}$ sequence and the biotinylated site. In this way, there was a satisfactory distance between MLL4 ${ }_{\text {Win }}$ and the sensor surface for either WDR5 or D92 $\mathrm{N}_{\text {WDR5 }}$ to interact without steric restriction from the BLI sensor. $5 \mathrm{nM}$ Biotinyl-(GGS) ${ }_{3} \mathrm{MLL}_{4} \mathrm{Win}_{\mathrm{NH}}$ was loaded onto the sensors for $15 \mathrm{~min}$. Washing off the unbound peptides was achieved by dipping the sensors into a peptide-free buffer for $5 \mathrm{~min}$. A 3 -fold serial dilution of either WDR 5 or 
D92 $\mathrm{N}_{\text {WDR5 }}$, which ranged between $0.1 \mu \mathrm{M}$ and $9 \mu \mathrm{M}$, was executed for inspecting the association process. Then, the BLI sensors were placed in a protein analyte-free buffer solution for inspecting the dissociation process. For all WDR5 and D92 $\mathrm{N}_{\text {WDR5 }}$ concentrations, the peptide-free BLI sensors were run in parallel as controls. These controls were used to subtract the baseline and drift in the sensorgrams. This process was required to extract the binding curves. All BLI experiments were conducted at $24^{\circ} \mathrm{C}$. For the fitting of binding curves, the FortéBio Octet Data Analysis software (FortéBio) was used. The curves generated by the association process were fitted using the following equation ${ }^{61}$ :

$$
Y=Y-\left(Y-Y_{0}\right) e^{-k_{\mathrm{obs}} t}
$$

Here, $Y_{0}$ and $Y_{\infty}$ are the response signals during the association process at zero and infinity times, respectively. $t$ is the cumulative time of the association reaction. $k_{\text {obs }}$ denotes the apparent first-order reaction rate constant of the association process. The curves generated by the dissociation process were fitted using the following equation:

$$
Y=Y+\left(Y_{0}-Y\right) e^{-k_{\text {off }} t}
$$

where, $Y_{0}$ and $Y_{\infty}$ are the response signals during the dissociation process at zero and infinity times, respectively. $k_{\text {off }}$ shows the dissociation rate constant. The association rate constant, $k_{\mathrm{on}}$, was included into the following function:

$$
k_{\mathrm{obs}}=k_{\mathrm{on}}[C]+k_{\mathrm{off}}
$$

where $[C]$ is the protein analyte concentration. Global fitting, which was conducted using several protein analyte concentrations, provided the corresponding $k_{\text {on }}$ and $k_{\text {off }}$ values. These kinetic parameters were employed to calculate the equilibrium dissociation constant, $K_{\mathrm{D}}$. Three independent BLI measurements were performed for quantitative kinetic determinations.

Simulation of the BLI response using results from single-channel electrical recordings. A model for multimodal protein recognition events was created using a competing-reaction method ${ }^{62}$. Each event was modelled as resulting from a different analyte competing to bind to the sensor. Consequently, the analytes were assumed to have the same masses and concentrations. The following differential equations were used to simulate the results:

$$
\begin{aligned}
& \frac{\mathrm{d} R_{1}}{\mathrm{~d} t}=k_{\text {on }-1}[C]\left(R_{\max }-R_{1}-R_{2}-R_{3}\right)-k_{\text {off }-1} R_{1} \\
& \frac{\mathrm{d} R_{2}}{\mathrm{~d} t}=k_{\text {on-2 }}[C]\left(R_{\max }-R_{1}-R_{2}-R_{3}\right)-k_{\text {off }-2} R_{2} \\
& \frac{\mathrm{d} R_{3}}{\mathrm{~d} t}=k_{\text {on-3 }}[C]\left(R_{\text {max }}-R_{1}-R_{2}-R_{3}\right)-k_{\text {off }-3} R_{3}
\end{aligned}
$$

Here $R_{1}, R_{2}$ and $R_{3}$ correspond to the BLI responses from short-, medium- and long-lived events, respectively. $[C]$ denotes the concentration of the protein analyte. $R_{\max }$ indicates the maximum BLI response determined by the total concentration of the immobilized partner. The total simulated BLI response at any given time, $R$, was given by:

$$
R=R_{1}+R_{2}+R_{3}
$$

Kinetic rate constants obtained from single-channel electrical recordings were plugged into these equations to obtain the expected BLI sensorgram. $R_{\max }$ was adjusted to match the simulated and experimental time-dependent BLI curves at equilibrium. A custom code and a mathematical algorithm were developed using MATLAB R2021a (MathWorks, Natick, MA).

Single-channel electrical recordings. Single-molecule electrophysiology was conducted using planar lipid bilayers ${ }^{99,63}$. A $25 \mu \mathrm{m}$-thick Teflon septum (Goodfellow Corporation, Malvern, PA) separated the two half sides of the chamber. A $90 \mu \mathrm{m}$-diameter aperture was created in this Teflon partition, which was then pretreated with hexadecane (Sigma-Aldrich, St. Louis, MO) dissolved in highly purified pentane (Fisher HPLC grade, Fair Lawn, NJ). A planar lipid bilayer was made of 1,2-diphytanoyl-sn-glycero-phosphatidylcholine (Avanti Polar Lipids, Alabaster, AL) across the small aperture. For all experiments, the buffer solution contained $300 \mathrm{mM} \mathrm{KCl}, 20 \mathrm{mM}$ Tris- $\mathrm{HCl}, 1 \mathrm{mM}$ TCEP, $\mathrm{pH}$ 7.5. Samples of protein nanopores and analytes were added to the cis compartment, which was grounded. The nanopore samples were added at a final concentration between 0.3 and $1 \mathrm{ng} / \mu \mathrm{l}$. An Axopatch 200B patch-clamp amplifier (Axon Instruments, Foster City, CA) was used to acquire single-channel electrical currents. The applied transmembrane potential was $-20 \mathrm{mV}$, unless otherwise stated. The electrical signal was low-pass filtered using an 8-pole Bessel filter (Model 900; Frequency Devices, Ottawa, IL) at a frequency of $10 \mathrm{kHz}$, then digitized using a low-noise acquisition system (Model Digidata $1440 \mathrm{~A}$; Axon Instruments) and sampled at a frequency of $50 \mathrm{kHz}$. For the analysis of binding events, single-channel electrical traces were filtered at a frequency of $1 \mathrm{kHz}$. All single-channel electrical recordings were acquired at room temperature $\left(23 \pm 1^{\circ} \mathrm{C}\right)$.
Statistical analysis of single-channel substate events. pClamp 10.7 (Axon Instruments) was used for data acquisition and analysis. ClampFit 10.7 (Axon) and Origin 8.6 (OriginLab, Northampton, MA) were used to produce figures. Different models were tested for both capture and release event durations. For each model, a kinetic rate matrix was employed to generate the probability distribution function $(\mathrm{PDF})$, so the kinetic rates were obtained by fitting the data using the maximum likelihood method ${ }^{35,36}$. A logarithm likelihood ratio (LLR) test was used to compare the results from different models and determine the number of statistically significant peaks that are best suited to the data ${ }^{37-39}$. At a confidence number of 0.95 , the best model for the release durations was a single-exponential fit. On the contrary, the best model for the capture durations was a three-exponential fit.

Molecular graphics. All cartoons that illustrate molecular graphics were prepared using the PyMOL Molecular Graphics System (Version 2.4.0 Schrödinger, LLC).

Reporting summary. Further information on research design is available in the Nature Research Reporting Summary linked to this article.

\section{Data availability}

In addition to Supplementary Information file, data supporting the findings of this article have been deposited in Zenodo at https://doi.org/10.5281/zenodo.5813135. The source data underlying Figs. 2e-i, 3a, b, 4e, f, 5d, e, and Supplementary Figs. 4, 8-10, 13, 14, 19, 21-25, and Supplementary Tables 4-7, 9-16, 19-23, \& 25-37 are provided in the Source data file. Entries $1 \mathrm{BY} 3$ and $4 \mathrm{ERZ}$ from the Protein Data Bank were used in this article for molecular visualizations. Source data are provided with this paper.

\section{Code availability}

All custom codes and mathematical algorithms used in this study are available at https:// doi.org/10.5281/zenodo.5813135.

Received: 16 July 2021; Accepted: 25 January 2022;

Published online: 21 February 2022

\section{References}

1. Zhang, C. \& Zhang, F. The multifunctions of WD40 proteins in genome integrity and cell cycle progression. J. Genomics 3, 40-50 (2015).

2. Afanasieva, E. et al. Structural diversity of oligomeric $\beta$-propellers with different numbers of identical blades. eLife 8, e49853 (2019).

3. Migliori, V., Mapelli, M. \& Guccione, E. On WD40 proteins: Propelling our knowledge of transcriptional control? Epigenetics 7, 815-822 (2012).

4. Jain, B. P. \& Pandey, S. WD40 repeat proteins: Signalling scaffold with diverse functions. Protein J. 37, 391-406 (2018).

5. Stirnimann, C. U., Petsalaki, E., Russell, R. B. \& Müller, C. W. WD40 proteins propel cellular networks. Trends Biochem. Sci. 35, 565-574 (2010)

6. Santosh Kumar, H. S., Kumar, V., Pattar, S. \& Telkar, S. Towards the construction of an interactome for Human WD40 protein family. Bioinformation 12, 54-61 (2016).

7. Schapira, M., Tyers, M., Torrent, M. \& Arrowsmith, C. H. WD40 repeat domain proteins: A novel target class? Nat. Rev. Drug Discov. 16, 773-786 (2017).

8. van Oijen, A. M. \& Dixon, N. E. Probing molecular choreography through single-molecule biochemistry. Nat. Struct. Mol. Biol. 22, 948-952 (2015).

9. Harrington, L., Cheley, S., Alexander, L. T., Knapp, S. \& Bayley, H. Stochastic detection of Pim protein kinases reveals electrostatically enhanced association of a peptide substrate. Proc. Natl. Acad. Sci. USA. 110, E4417-E4426 (2013).

10. Harrington, L., Alexander, L. T., Knapp, S. \& Bayley, H. Single-molecule protein phosphorylation and dephosphorylation by nanopore enzymology. ACS Nano. 13, 633-641 (2018).

11. Ying, Y. L., Yu, R. J., Hu, Y. X., Gao, R. \& Long, Y. T. Single antibody-antigen interactions monitored via transient ionic current recording using nanopore sensors. Chem. Commun. (Camb.) 53, 8620-8623 (2017).

12. Thakur, A. K. \& Movileanu, L. Real-time measurement of protein-protein interactions at single-molecule resolution using a biological nanopore. Nat Biotechnol. 37, 96-101 (2019).

13. Thakur, A. K. \& Movileanu, L. Single-molecule protein detection in a biofluid using a quantitative nanopore sensor. ACS Sens. 4, 2320-2326 (2019)

14. Wei, R., Gatterdam, V., Wieneke, R., Tampe, R. \& Rant, U. Stochastic sensing of proteins with receptor-modified solid-state nanopores. Nat. Nanotechnol. 7, 257-263 (2012).

15. Sackmann, B. \& Neher, E. Single-Channel Recording, Edn. Second Edition. (Kluwer Academic/Plenum Publishers, New York; 1995).

16. Weichbrodt, C. et al. Antibiotic translocation through porins studied in planar lipid bilayers using parallel platforms. Analyst 140, 4874-4881 (2015).

17. Restrepo-Perez, L., Joo, C. \& Dekker, C. Paving the way to single-molecule protein sequencing. Nat. Nanotechnol. 13, 786-796 (2018). 
18. Alfaro, J. A. et al. The emerging landscape of single-molecule protein sequencing technologies. Nat. Methods 18, 604-617 (2021).

19. Fahie, M. A., Yang, B., Mullis, M., Holden, M. A. \& Chen, M. Selective detection of protein homologues in serum using an OmpG nanopore. Anal. Chem. 87, 11143-11149 (2015).

20. Galenkamp, N. S., Soskine, M., Hermans, J., Wloka, C. \& Maglia, G. Direct electrical quantification of glucose and asparagine from bodily fluids using nanopores. Nat. Commun. 9, 4085 (2018).

21. Lucas, F. L. R. et al. Automated electrical quantification of Vitamin B1 in a bodily fluid using an engineered nanopore sensor. Angew. Chem. Int. Ed. Engl. 60, 22849-22855 (2021).

22. Ayub, M. \& Bayley, H. Engineered transmembrane pores. Curr. Opin. Chem. Biol. 34, 117-126 (2016).

23. Burns, J. R., Seifert, A., Fertig, N. \& Howorka, S. A biomimetic DNA-based channel for the ligand-controlled transport of charged molecular cargo across a biological membrane. Nat. Nanotechnol. 11, 152-156 (2016).

24. Howorka, S. Building membrane nanopores. Nat. Nanotechnol. 12, 619-630 (2017).

25. Varongchayakul, N., Song, J., Meller, A. \& Grinstaff, M. W. Single-molecule protein sensing in a nanopore: A tutorial. Chem. Soc. Rev. 47, 8512-8524 (2018).

26. Sun, J., Thakur, A. K. \& Movileanu, L. Protein ligand-induced amplification in the 1/f noise of a protein-selective nanopore. Langmuir 36, 15247-15257 (2020).

27. Schmid, S. \& Dekker, C. Nanopores: A versatile tool to study protein dynamics. Essays Biochem. 65, 93-107 (2021).

28. Locher, K. P. et al. Transmembrane signaling across the ligand-gated FhuA receptor: Crystal structures of free and ferrichrome-bound states reveal allosteric changes. Cell 95, 771-778 (1998).

29. Denesyuk, A., Denessiouk, K. \& Johnson, M. S. Top surface blade residues and the central channel water molecules are conserved in every repeat of the integrin-like $\beta$-propeller structures. J. Struct. Biol. 201, 155-161 (2018).

30. Patel, A., Dharmarajan, V. \& Cosgrove, M. S. Structure of WDR5 bound to mixed lineage leukemia protein-1 peptide. J. Biol. Chem. 283, 32158-32161 (2008).

31. Song, J. J. \& Kingston, R. E. WDR5 interacts with mixed lineage leukemia (MLL) protein via the histone H3-binding pocket. J. Biol. Chem. 283, 35258-35264 (2008).

32. Dharmarajan, V., Lee, J. H., Patel, A., Skalnik, D. G. \& Cosgrove, M. S. Structural basis for WDR5 interaction (Win) motif recognition in human SET1 family histone methyltransferases. J. Biol. Chem. 287, 27275-27289 (2012).

33. Zhang, P., Lee, H., Brunzelle, J. S. \& Couture, J. F. The plasticity of WDR5 peptide-binding cleft enables the binding of the SET1 family of histone methyltransferases. Nucleic Acids Res. 40, 4237-4246 (2012).

34. Mohammad, M. M., Howard, K. R. \& Movileanu, L. Redesign of a plugged beta-barrel membrane protein. J. Biol. Chem. 286, 8000-8013 (2011).

35. Colquhoun, D. \& Sigworth, F. J. in Single-channel recording, 2nd. edn. (ed. B. Sackmann, Neher, E.) 483-587 (Plenum Press, New York; 1995).

36. Colquhoun, D., Hatton, C. J. \& Hawkes, A. G. The quality of maximum likelihood estimates of ion channel rate constants. J. Physiol. 547, 699-728 (2003).

37. McManus, O. B., Blatz, A. L. \& Magleby, K. L. Sampling, log binning, fitting, and plotting durations of open and shut intervals from single channels and the effects of noise. Pflug. Arch. 410, 530-553 (1987).

38. McManus, O. B. \& Magleby, K. L. Kinetic states and modes of single largeconductance calcium-activated potassium channels in cultured rat skeletalmuscle. J. Physiol. (Lond.) 402, 79-120 (1988).

39. Couoh-Cardel, S., Hsueh, Y. C., Wilkens, S. \& Movileanu, L. Yeast V-ATPase proteolipid ring acts as a large-conductance transmembrane protein pore. Sci. Rep. 6, 24774 (2016).

40. Tate, J. G. et al. COSMIC: The catalogue of somatic mutations in cancer. Nucleic Acids Res. 47, D941-d947 (2019).

41. Mobley, D. L. \& Dill, K. A. Binding of small-molecule ligands to proteins: "what you see" is not always "what you get". Structure 17, 489-498 (2009).

42. Imran, A. et al. Kinetics of the multitasking high-affinity Win binding site of WDR5 in restricted and unrestricted conditions. Biochem. J. 478, 2145-2161 (2021).

43. Paul, F. et al. Protein-peptide association kinetics beyond the seconds timescale from atomistic simulations. Nat. Commun. 8, 1095 (2017).

44. Stadmiller, S. S., Aguilar, J. S., Parnham, S. \& Pielak, G. J. Protein-peptide binding energetics under crowded conditions. J. Phys. Chem. B 124, 9297-9309 (2020).

45. Bikwemu, R., Wolfe, A. J., Xing, X. \& Movileanu, L. Facilitated translocation of polypeptides through a single nanopore. J. Phys.: Condens. Matter 22, 454117 (2010).

46. Ho, C. W. et al. Engineering a nanopore with co-chaperonin function. Sci. Adv. 1, e1500905 (2015).

47. Nivala, J., Marks, D. B. \& Akeson, M. Unfoldase-mediated protein translocation through an alpha-hemolysin nanopore. Nat. Biotechnol. 31, 247-250 (2013).
48. Di Trani, J. M. et al. Rapid measurement of inhibitor binding kinetics by isothermal titration calorimetry. Nat. Commun. 9, 893 (2018).

49. Douzi, B. Protein-Protein Interactions: Surface Plasmon Resonance. Methods Mol. Biol. 1615, 257-275 (2017).

50. Guarnaccia, A. D. et al. Impact of WIN site inhibitor on the WDR5 interactome. Cell Rep. 34, 108636 (2021).

51. Alicea-Velázquez, N. L. et al. Targeted Disruption of the Interaction between WD-40 Repeat Protein 5 (WDR5) and Mixed Lineage Leukemia (MLL)/SET1 Family Proteins Specifically Inhibits MLL1 and SETd1A Methyltransferase Complexes. J. Biol. Chem. 291, 22357-22372 (2016).

52. Dou, Y. et al. Regulation of MLL1 H3K4 methyltransferase activity by its core components. Nat. Struct. Mol. Biol. 13, 713-719 (2006)

53. Patel, A., Dharmarajan, V., Vought, V. E. \& Cosgrove, M. S. On the mechanism of multiple lysine methylation by the human mixed lineage leukemia protein-1 (MLL1) core complex. J. Biol. Chem. 284, 24242-24256 (2009).

54. Shinsky, S. A., Monteith, K. E., Viggiano, S. \& Cosgrove, M. S. Biochemical reconstitution and phylogenetic comparison of human SET1 family core complexes involved in histone methylation. J. Biol. Chem. 290, 6361-6375 (2015).

55. Guarnaccia, A. D. \& Tansey, W. P. Moonlighting with WDR5: A Cellular Multitasker. J. Clin. Med. 7, 21 (2018).

56. Bryan, A. F. et al. WDR5 is a conserved regulator of protein synthesis gene expression. Nucleic Acids Res 48, 2924-2941 (2020).

57. Thomas, L. R., Adams, C. M., Fesik, S. W., Eischen, C. M. \& Tansey, W. P. Targeting MYC through WDR5. Mol. Cell. Oncol. 7, 1709388 (2020).

58. Jiang, $H$. The complex activities of the SET1/MLL complex core subunits in development and disease. Biochim. Biophys. Acta Gene. Regul. Mech. 1863, 194560 (2020).

59. Kudlinzki, D., Schmitt, A., Christian, H. \& Ficner, R. Structural analysis of the C-terminal domain of the spliceosomal helicase Prp22. Biol. Chem. 393, 1131-1140 (2012).

60. Patel, A., Vought, V. E., Dharmarajan, V. \& Cosgrove, M. S. A conserved arginine-containing motif crucial for the assembly and enzymatic activity of the mixed lineage leukemia protein-1 core complex. J. Biol. Chem. 283, 32162-32175 (2008).

61. Movileanu, L., Cheley, S., Howorka, S., Braha, O. \& Bayley, H. Location of a Constriction in the Lumen of a Transmembrane Pore by Targeted Covalent Attachment of Polymer Molecules. J. Gen. Physiol. 117, 239-251 (2001).

62. Karlsson, R. \& Fält, A. Experimental design for kinetic analysis of proteinprotein interactions with surface plasmon resonance biosensors. J. Immunol. Methods 200, 121-133 (1997).

63. Larimi, M. G., Mayse, L. A. \& Movileanu, L. Interactions of a Polypeptide with a Protein Nanopore Under Crowding Conditions. ACS nano 13, 4469-4477 (2019).

\section{Acknowledgements}

We thank our colleagues in the Movileanu and Cosgrove laboratories and at Ichor Life Sciences laboratories for their comments on the manuscript and stimulating discussions as well as for their technical assistance during the very early stage of this project. This work was supported by the National Cancer Institute of the U.S. National Institutes of Health grant R01 CA140522 (to M.S.C) and by the National Institute of General Medical Sciences of the U.S. National Institutes of Health grant R01 GM129429 (to L.M.).

\section{Author contributions}

L.A.M., A.I., M.S.C., A.J.W., and L.M. designed research. L.A.M. and A.I. performed research and analyzed data. M.G.L., M.S.C., and L.M. supervised research. L.A.M. and L.M. wrote the paper.

\section{Competing interests}

The authors declare no competing interests.

\section{Additional information}

Supplementary information The online version contains supplementary material available at https://doi.org/10.1038/s41467-022-28465-8.

Correspondence and requests for materials should be addressed to Liviu Movileanu.

Peer review information Nature Communications thanks Chan Cao and the other, anonymous, reviewer(s) for their contribution to the peer review of this work.

Reprints and permission information is available at http://www.nature.com/reprints

Publisher's note Springer Nature remains neutral with regard to jurisdictional claims in published maps and institutional affiliations. 
(c) (i) Open Access This article is licensed under a Creative Commons Attribution 4.0 International License, which permits use, sharing, adaptation, distribution and reproduction in any medium or format, as long as you give appropriate credit to the original author(s) and the source, provide a link to the Creative Commons license, and indicate if changes were made. The images or other third party material in this article are included in the article's Creative Commons license, unless indicated otherwise in a credit line to the material. If material is not included in the article's Creative Commons license and your intended use is not permitted by statutory regulation or exceeds the permitted use, you will need to obtain permission directly from the copyright holder. To view a copy of this license, visit http://creativecommons.org/ licenses/by/4.0/.

(C) The Author(s) 2022 